\title{
Angiotensin-Converting Enzyme Type 2 as a Molecular Mediator for Infection of Cells with SARS-CoV and SARS-CoV-2 Viruses
}

\author{
A. O. Shpakov
}

\begin{abstract}
Translated from Rossiiskii Fiziologicheskii Zhurnal imeni I. M. Sechenova, Vol. 106, No. 7, pp. 795-810, July, 2020. Original article submitted April 10, 2020. Revised version received April 21, 2020. Accepted April 21, 2020.
\end{abstract}

Penetration of coronavirus SARS-CoV-2 (Severe Acute Respiratory Syndrome Coronavirus 2), the cause of the COVID-19 pandemic, into cells requires interaction of the surface spike S protein of the virus with extracellular domain of the membrane-bound form of angiotensin-converting enzyme type 2 (ACE2). This enzyme, the key component of the renin-angiotensin system, is responsible for the synthesis of angiotensin-(1-7), which has vasodilator and autoimmune properties, from angiotensin II, a powerful vasoconstrictor, whose synthesis is catalyzed by angiotensin-converting enzyme (ACE), a functional antagonist of ACE2. After site-specific hydrolysis by transmembrane serine protease TMPRSS2 in infected cells, viral $S$ protein specifically binds ACE2, which is the trigger for internalization of the virus into cells by endocytosis. Suppression of this process with drugs inhibiting TMPRSS2 protease and impairing the interaction of $\mathrm{S}$ protein with ACE2 prevents infection and is therefore a potential approach to the treatment and prophylaxis of COVID-19. This mechanism of penetration into cells is also used by SARS-CoV virus, the pathogen of atypical pneumonia, which is related to SARS-CoV-2. Treatment of patients with arterial hypertension and diabetes mellitus using ACE inhibitors and angiotensin receptor blockers, statins, and various antidiabetic drugs generally increases the expression and activity of ACE2, which increases the risk of infection with SARS-CoV-2 and worsens the outcome of disease. During the process of infection, the virus forms a complex with ACE2 and decreases the quantity of ACE2 on the cell surface, impairing ACE2-dependent physiological processes, and this is one of the causes of acute respiratory distress syndrome and heart failure in COVID-19 patients. The present review analyzes the functional role of ACE2 in the infection of cells by SARS-CoV-2 and SARS-CoV, and the molecular mechanisms of this process and its pathophysiological consequences are discussed.

Keywords: angiotensin-converting enzyme type 2, renin-angiotensin system, TTMPRSS2 protease, SARS-CoV-2, SARS-CoV, ADAM17 protease, angiotensin-converting enzyme inhibitor, angiotensin receptor type 1 blocker.

Introduction. A new coronavirus was detected in Wuhan City (China) in December 2019 and this then spread explosively worldwide. On February 11, 2020 the pathogen of this infection was classified by the World Health Organization as SARS-CoV-2 (Severe Acute Respiratory Syndrome Coronavirus 2), and the disease itself was named

Sechenov Institute of Evolutionary Physiology and Biochemistry, Russian Academy of Sciences, St. Petersburg, Russia;

e-mail: alex_shpakov@list.ru.
COVID-19 [1]. Coronavirus SARS-CoV-2 is $30 \mathrm{~kb}$ in size and is a single-stranded RNA virus belonging to the Coronaviridae family. The genome encodes four structural proteins - membrane protein $\mathrm{M}$, coat protein $\mathrm{E}$, nucleocapsid protein $\mathrm{N}$, and glycoprotein spike protein $\mathrm{S}$, which protrudes from the surface of the viral capsid forming a spikelike structure which makes contact with the host cell surface [2]. The acute respiratory infection produced by SARSCoV-2 is characterized by relatively high lethality among elderly people (over 60 years of age) and among patients 


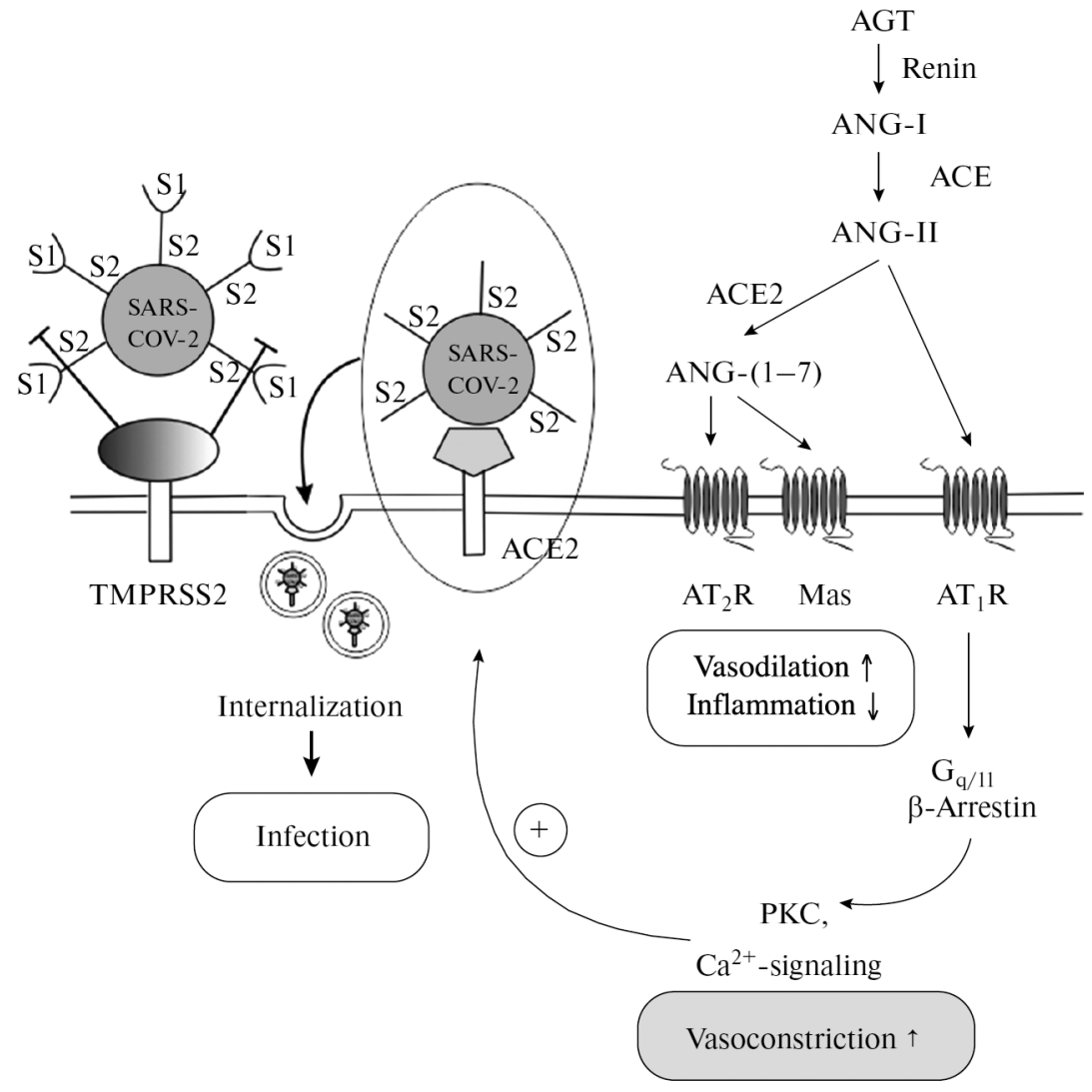

Fig. 1. The role of TMPRSS23, ACE2, and components of the angiotensin pathway in penetration of SARS-CoV-2 virus into cells. TMPRSS2 - transmembrane protease, serine 2; ACE - angiotensin-converting enzyme; ACE2 - angiotensin-converting enzyme type 2; AT1R - type 1 angiotensin-II receptor; AGT - angiotensinogen; ANG-I - angiotensin-I; ANG-II - angiotensin-II; ANG-(1-7) - angiotensin-(1-7); $\mathrm{G}_{\mathrm{q} / 11}-$ heterotrimeric $\mathrm{G}_{\mathrm{q} / 11}$ protein ; PKC - protein kinase $\mathrm{C} ; \mathrm{S} 1, \mathrm{~S} 2$ - subdomains $\mathrm{S} 1$ and $\mathrm{S} 2$ of the viral surface spike $\mathrm{S}$ protein.

with cardiovascular disease, kidney disease, diabetes mellitus, oncological and autoimmune diseases [3-6]. Working from current epidemiological data, there are grounds for the view that the severity of symptoms and the level of lethality of SARS-CoV-2 infections are no greater than those caused by the related virus SARS-CoV, which was responsible for the 2003 epidemic. However, the wide dissemination of SARS-CoV-2 - now a pandemic - along with the faster human-to-human transmission of SARS-CoV-2 than SARS$\mathrm{CoV}$ and its relatively high reproduction number create high risks to people's health in all countries. This gave specialists in medicine, biology, and related sciences an extremely short period of time to identify the molecular mechanisms of infection of cells by SARS-CoV-2 and to develop effective approaches to treating COVID-19.

There is great interest in data showing that effective penetration of SARS-CoV-2 into infected cells requires the "assistance" of one of the key enzymes of the renin-angiotensin system - angiotensin-converting enzyme type 2 (ACE2). This is in good agreement with the fact that COVID-19 is most severe in elderly people and patients with chronic heart and kidney disease and diabetes mellitus, which have long been treated with anti-hypertensive and antidiabetic drugs. These patients show larger changes in
ACE2 expression and activity, which generally increase as a result of the drug treatment used $[7,8]$. This has led to extensive discussion of how to address variation in ACE2 expression levels in patients with diabetes and cardiovascular disease in assessments of their risk of infection with SARSCoV-2, predicting the course and outcome of COVID-19, and evaluating how treatment of these patients with angiotensin-converting enzyme (ACE) inhibitors, type 1 angiotensin II receptor blockers (AT1R), statins, and antidiabetics might influence ACE2-mediated SARS-CoV-2 virus infection. It should be noted that SARS-CoV, which has features in common with SARS-CoV-2, also uses ACE2 for host cell penetration [9-11]. This indicates that developments in the interaction of SARS-CoV virus with ACE2 made previously, long before the COVID-19 pandemic, could be used to decipher the molecular mechanisms of cell penetration by the new coronavirus, SARS-CoV-2, and have been adopted by virologists and molecular biologists seeking to slow the COVID-19 pandemic $[12,13]$. The present review also addresses these issues. However, we will start with a brief discussion of the structural-functional organization of the renin-angiotensin system and the structure and regulatory properties of angiotensin receptors and the underlying signal cascades and targets. 
The Renin-Angiotensin System and Angiotensin Receptors. The renin-angiotensin system includes enzymes with proteolytic activity which are involved in the interconversion of peptides of the angiotensin family, their receptors, and their associated intracellular signal cascades and effectors (Fig. 1).

The first stage in converting peptides of the angiotensin family is mediated by renin, a proteolytic enzyme which cleaves an $\mathrm{N}$-terminal decapeptide, i.e., angiotensin I (ANG-I) from the angiotensinogen molecule (AGT), which is 153 amino acids long [14]. AGT is a member of the serine protease family (serpines), and AGT activity depends largely on its level of glycosylation [15]. The enzyme ACE converts ANG-1 to ANG-II and is a membrane-bound zinc- and chloride-dependent dipeptidylcarboxypeptidase (E.C. 3.4.15.1), whose catalytic site is located in the extracellular space. This enzyme is the target of many ACE inhibitors, whose pharmacological effects are directed to weakening arterial hypertension [16].

Exopeptidase ACE2 (E.C. 3.4.17.23), which converts ANG-II to ANG-(1-7) and ANG-I to ANG-(1-9), despite a notable level of homology with the enzyme ACE (about $40 \%$ identical and $60 \%$ physicochemically similar amino acid residues), has an opposite functional activity, as it prevents the vasoconstrictor effects of ANG-II and, conversely, increases the countering vasodilatory effects of peptides ANG-(1-7) and ANG-(1-9) [17-20]. In contrast to ACE, ACE2 is a monocarboxypeptidase and has relatively low specificity, hydrolyzing not only angiotensin peptides, but also apelin, opioid peptides, and kinins. ACE inhibitors do not affect the catalytic activity of ACE2, so the catalytic and allosteric sites which are the targets of these inhibitors are significantly different in the ACE and ACE2 molecules. There are two forms of ACE2 - the functionally active membrane-bound form which, along with the N-terminal catalytic ectodomain, has a transmembrane domain and a small cytoplasmic domain, and a soluble form corresponding to the $\mathrm{N}$-terminal ectodomain, which is generated by cleavage of the extracellular part of the ACE2 molecule from its transmembrane domain [17]. This process is mediated by the quite large membrane-bound protease ADAM17 [21-23]. ADAM17 protease is a type I transmembrane protein and includes a number of functional domains 1) the N-terminal domain, which functions as an "internal" blocker of proteas activity; 2) a metalloprotease (catalytic) domain 3) a domain proximal to the membrane domain enriched with cysteine residues; 5) a transmembrane domain; and 6) a small cytoplasmic segment [24]. In terms of topology in the plasma membrane, the two enzymes, ACE2 and ADAM17, are similar. Relatively high levels of expression of ACE2 and ADAM17 are seen in the heart, lungs, kidneys, brain, and testes, while levels are lower or insignificant in other tissues. These data indicate nonuniform distributions of the expression and activity of these enzymes in different organs and tissues and a close interaction between them $[17,24]$. It should, however, be noted that ADAM17 protease, like ACE2, controls the activity of a multitude of other proteins which are components of signal and effector systems [24].

The main targets of ANG-II are two subtypes of G-protein-coupled receptors - AT1R and AT2R, which are very different both in terms of the spectrum of signal cascades regulated by them and in terms of physiological responses [25-27]. AT2R may modulate the functional activity of AT1R, forming heterodimer receptor complexes with them $[28,29]$, and they also suppress the activity of the postreceptor components of AT1R-activated signal cascades [30].

Binding of AT1R with agonists leads to stimulation of heterotrimeric $\mathrm{G}_{\mathrm{q} / 11}$ protein, which activates phosphoinositol-specific phospholipase $\mathrm{C} \beta$. This results in formation of inositol-1,4,5-triphosphate, which, activating specific intracellular receptors, stimulates $\mathrm{Ca}^{2+}$ release from intracellular depots and synthesis of diacylglycerol, which activates phorbol-sensitive isoforms of protein kinase $\mathrm{C}$ (PKC) [26, 31]. In addition, AT1R agonists activate regulatory proteins $\beta$-arrestins, stimulating a cascade of mitogen-activated protein kinases (MAPK) and a number of other $\beta$-arrestin-dependent pathways [26, 32]. It is important to note that ANG-II-induced activation of ERK1/2, the key components of the MAPK cascade, can occur with the involvement of $\mathrm{G}_{\mathrm{q} / 11}$ proteins [33] and in a G-protein-independent manner, via activation of $\beta$-arrestins [32, 34]. In contrast to AT1R, AT2R receptors exert their actions via pathways independent of $G$ proteins and $\beta$-arrestins, which is not typical of "classical" G-protein-coupled receptors. Its effects, as noted above, can be realized via formation of heterodimer complexes with AT1R, Mas receptors, and other G-proteincoupled receptors. In addition, being activated by ANG-II, AT2R receptors can operate via a noncanonical cascade to increase the activity of various NO synthase isoforms, thus activating NO-dependent pathways and stimulating the MAPK and 3-phosphoinositide pathways [27, 29, 35, 36].

Angiotensin peptides ANG-(1-7) and its truncated ana$\log$ ANG-(1-5) interact specifically with G-protein-coupled Mas receptors, which are the product of the MAS1 oncogene, through which they have anti-inflammatory, vasodilator, and antifibrotic actions on their target cells [29, 37]. While Mas receptors are functional antagonists of AT1R, their main ligand is the peptide ANG-(1-7) - a functional antagonist of ANG-II. ANG-(1-7) peptide can be produced by hydrolytic cleavage of ANG-I or ANG-(1-9) by neprilysin, though the main pool is produced by ACE2-catalyzed ANG-II hydrolysis [16].

The ACE2-Mediated Mechanism of Penetration of SARS-CoV Virus into Cells. As already noted, before the appearance of SARS-CoV-2, studies of the related virus SARS-CoV, which causes acute respiratory syndrome, demonstrated the ability of this latter virus to use the membrane-bound form of ACE2 to enter host cells [9-11]. After establishment of the interaction between SARS-CoV pene- 
tration into human lung cells bearing ACE2, a proteolytic enzyme was found which was required for this penetration. This was transmembrane serine protease TMPRSS2 (transmembrane protease, serine 2), which is expressed at high intensity in epithelial cells in human lungs and promotes their infection with influenza type A virus and metapneumovirus [38-41]. Studies of influenza virus type A have shown that TMPRSS2 protease cleaves the coat glycoprotein hemagglutinin, with the result that influenza A acquires the ability to fuse with the cell membrane and then undergo endocytosis to enter the cell $[38,40]$. In the case of SARS-CoV, TMPRSS2 protease partially hydrolyzes the viral surface spike protein $\mathrm{S}$, thus priming it to induce effective fusion of the virus with the plasma membrane and internalization into the host cell [10]. It should be noted that virus replication in cells with elevated TMPRSS2 protease expression is not accompanied by any increase in the ability of the virus to infect or any alteration in the structure of viral protein $\mathrm{S}$, indicating the involvement of TMPRSS2 protease in the process of viral entry into cells across the cell membrane and not in controlling its subsequent replication [10].

Further studies showed that TMPRSS2 protease can cleave and activate not only viral protein $\mathrm{S}$, but also the membrane form of ACE2, which also makes a contribution to the penetration of SARS-CoV virus into the cell. Cleavage occurs at lysine and arginine residues in the segment 697-716 of ACE2. Sites targeted by TMPRSS2 protease differ from those at which the enzyme is cleaved by transmembrane ectoprotease ADAM17, with the result that the consequences of this cleavage are different. This TMPRSS2-induced cleavage of ACE2 promotes infection of cells by virus, while cleavage of the enzyme by ADAM17, conversely, suppresses this process [11].

An English group demonstrated in 2005 that cleavage of ACE2 by ADAM17 protease produces a soluble form of ACE2, which specifically binds surface $S$ protein of SARS$\mathrm{CoV}$ virus, thus preventing its interaction with the membrane form of ACE2 involved in target cell infection [21]. Small interfering RNA inhibiting ADAM17 synthesis suppresses cleavage of ACE2, while increases in ADAM17 activity, conversely, stimulate it. In HEK-293 and Huh-7 cells expressing membrane-bound ACE2, phorbol ester - phorbol-12-myristate acetate - stimulates ADAM17-induced cleavage of the ACE2 molecule; selective metalloproteinase inhibitors TAPI-1 and GM6001 prevent this process. These data provide evidence that the process of activation of ADAM17 involves signal pathways mediated by phorbol-sensitive isoforms of PKC [21]. Subsequent studies showed that neurons and glial cells in the brains of patients with hypertension, which show elevated ACE2 expression and activity, also show increases in ADAM17 protease. ADAM17 activity, and, thus, the accumulation of soluble forms of ACE2, increases in ANG-II-induced stimulation of AT1R receptors [22]. All these points indicate that ANG-II and other AT1R agonists stimulate the signal pathway in- cluding AT1R, $\mathrm{G}_{\mathrm{q} / 11}$ protein, and phospholipase $\mathrm{C} \beta$, resulting in an increase in diacylglycerol production, activation of phorbol-sensitive isoforms of PKC, and intensification of ADAM17-induced ACE2 cleavage [23]. There are grounds for taking the view that other activators of these PKC isoforms can also promote stimulation of ADAM17 and induce hydrolysis of membrane-bound ACE2.

With the aim of deciphering the mechanisms of interaction of SARS-CoV protein $\mathrm{S}$ with the membrane-bound form of ACE2, the key amino acid residues involved in this process were identified. These studies established that the most important residues for interaction with human ACE2 are $\mathrm{Tyr}^{442}, \mathrm{Leu}^{472}, \mathrm{Asn}^{479}, \mathrm{Asp}^{480}, \mathrm{Thr}^{487}$, and $\mathrm{Tyr}^{491}$ of SARS-CoV viral S protein (NP_828851.1). These amino acid residues are part of the so-called "receptor"-binding domain, corresponding to the sequence $424-494$ of protein S [42, 43]. In turn, highly conserved amino acid residues $\mathrm{Lys}^{31}, \mathrm{Glu}^{35}, \mathrm{Asp}^{38}, \mathrm{Met}^{82}$, and $\mathrm{Lys}^{353}$, four of which are located at the N-terminal part of the ectodomain of the enzyme responsible for the interaction with viral protein, were identified in the structure of ACE2 [43].

A set of studies led to the entirely grounded suggestion that suppression of TMPRSS2/ACE2-mediated penetration of SARS-CoV virus into cells may be an effective route to preventing acute respiratory infections caused by this coronavirus $[10,11]$. Certain hopes were also linked with regulation of the functional activity of transmembrane protease ADAM17, which, in contrast to TMPRSS2 protease, prevents infection of cells by SARS-CoV. However, the end of the epidemic and reassessment of the prospects for developing vaccines against SARS-CoV prevented continuation of these studies. At the same time, existing progress provided practical and theoretical grounds for studying ACE2-mediated mechanisms of cell infection by the novel virus SARS-CoV-2.

ACE2-Mediated Mechanism of Penetration of SARS-CoV Virus into Cells. Considering the structural and functional similarities of SARS-CoV and SARS-CoV-2 viruses apparent at the very beginning of studies started at the beginning of 2020, most attention has been focused on the role of ACE2 and TMPRSS2 and ADAM17 proteases in the molecular mechanisms of the penetration of SARSCoV-2 into cells. The molecular bases of the interaction of ACE2 with the superficial viral S protein of SARS-CoV-2 and the role of the transmembrane proteases TMPRSS23 and ADAM17 in this process, these being able to alter the structure of S protein (TMPRSS2) and induce hydrolytic cleavage of the membrane-bound form of ACE2 (ADAM17, TMPRSS2), have been studied [7, 12, 13, 44-49].

Superficial S proteins of SARS-CoV and SARS-CoV-2 viruses have significant primary structural similarities $(76 \%$ of the amino acid residues are identical), including in the "receptor"-binding domain of these proteins. Five of the six amino acid residues playing the key role in the interaction of SARS-Cov S protein with the membrane-bound form of 
ACE2 were altered in the structure of SARS-CoV-2 S protein (PY_009724390.1) (Leu ${ }^{455}, \mathrm{Phe}^{486}, \mathrm{Gln}^{493}, \mathrm{Ser}^{494}$, and $\mathrm{Asn}^{501}$ ), the exception being $\mathrm{Tyr}^{505}$ of the SARS-CoV-2 S protein, which corresponds to $\mathrm{Tyr}^{491}$ of the SARS-CoV S protein. Despite this fact, the topology and conformational characteristics of the ACE2-binding site of the S proteins of both viruses are similar [45]. Molecular modeling data indicate that the greatest affinity for SARS-CoV-2 S protein is seen with ACE2 from humans, pangolins, dogs, cats, and golden hamsters, while ACE2 from, for example, mice and rats interacts weakly with this protein. In this model, the distance between the Phe ${ }^{486}$ of the SARS-CoV-2 S protein and the $\mathrm{Met}^{82}$ residue of human ACE2 in complexes of these proteins is $3.753 \AA$. The models of the SARS-CoV-2 $\mathrm{S}$ protein with ACE2 from pangolins, dogs, cats, and golden hamsters give corresponding distances of 1.621, 2.695, 3.753 , and $2.024 \AA$, respectively, which may point to the ability for effective penetration of cells in these animals. The ability of ACE2 to interact effectively with SARS$\mathrm{CoV}-2 \mathrm{~S}$ protein is largely due to the nature of the amino acid residue at position 82 . Theoretical analysis shows that mutant human ACE2 with substitution of the methionine residue in this position with asparagine increased the penetrating ability of SARS-CoV-2 into cells, as compared with wild-type ACE2 [45]. Other authors, working on the results of their structural analysis of ACE2 and the interaction of this enzyme with SARS-CoV-2 S protein, showed that as in humans, this interaction can take place in various other mammals (pangolins, pigs, sheep, cats, dogs, and others) which have forms of ACE2 able to undergo highly effective interactions with viral $\mathrm{S}$ protein of SARS-CoV-2. The results provided evidence supporting a high probability that these animals can be infected with SARS-CoV-2 virus. The exceptions are mice and rats, which creates limitations to their use in experiments addressing SARS-CoV-2 [47].

Studies have shown that as in the case of SARS-CoV, TMPRSS2 protease plays an exclusively important role in the ACE2-mediated penetration of SARS-CoV-2 virus into target cells $[6,13,46]$. S protein is also a target for TMPRSS2, and is cleaved by the protease at two sites. One is enriched with arginine residues and is located at the boundary of the two subdomains, S1 and S2, such that it is designated $\mathrm{S} 1 / \mathrm{S} 2$, while the other is located in the $\mathrm{N}$-terminal part of the S2 domain and is therefore termed the S2 site. It should be noted that the $\mathrm{S} 1$ subdomain contains the "receptor"-binding region and is responsible for attachment to membrane-bound ACE2, while subdomain S2 is responsible for forming complexes with the ectodomain of ACE2 and the subsequent penetration of virus particles into cells. Proteolysis at the S1/S2 site occurs much more intensely than at the $\mathrm{S} 2$ site, and plays an exclusively important role in penetration of virus into cells. This is evidenced by data on the large quantity of virus $\mathrm{S}$ protein cleaved at the $\mathrm{S} 1 / \mathrm{S} 2$ site within cells infected with SARS-CoV-2. Cleavage of viral $\mathrm{S}$ protein allows the $\mathrm{S} 2$ subunit to be released from its complex with the $\mathrm{S} 1$ subdomain, after which it acquires the ability to interact effectively with the ectodomain of ACE2 and form ACE2-S2 (SARS-CoV-2) complexes, promoting internalization of virus into the cell by endocytosis $[13,50]$. It is important to note that the S1/S2 cleavage site is absent from the $S$ proteins of other viruses, including the $S$ protein of RaTG13 virus, which is phylogenetically closest to SARS-CoV-2. This indicates that TMPRSS2 can mediate the penetration of SARS-CoV-2 into host cells but, as suggested, may be ineffective in relation to atypical pneumonia virus SARS-CoV and various other coronaviruses related to SARS-CoV-2 [13]. VeroE6 cell cultures, in which TMPRSS2 is expressed, had high ability to be infected by SARS-CoV-2, while in the absence of protease, virus did not enter cells and infection did not occur [46].

Along with TMPRSS2, cathepsins B and L, which are endosomal cysteine proteases, can also cleave coronavirus $\mathrm{S}$ proteins [51]. However, in the case of SARS-CoV and various other related viruses, this does not lead to "correct" priming of $\mathrm{S}$ protein and penetration of virus particles into cells $[50,52,53]$, and in the case of SARS-CoV-2, it has been suggested to make only a minor contribution to this process [13]. This is indicated by data showing that SARSCoV-2 infection of Calu-3 cells treated with camostat mesilate, a selective TMPRSS2 inhibitor, was suppressed but not completely blocked. This may be due to priming of $\mathrm{S}$ protein by cathepsins $\mathrm{B}$ and $\mathrm{L}$, though incomplete inhibition of TMPRSS2 cannot be excluded [13]. In this regard, we must note that some contribution to the entry of Middle East Respiratory Syndrome CoV virus into cells (MERS$\mathrm{CoV}$ ) is made by the serine protease furin, though effective infection also requires TMPRSS 2 protease $[54,55]$. All these points provide evidence that camostat mesilate and other TMPRSS2 protease inhibitors are of significant interest for developing drugs to prevent and treat coronavirus infections, all the more so given that camostat mesilate has already undergone clinical trials and has been approved for use as a drug [52, 53].

As regards the role of ADAM17 protease in infection by SARS-CoV-2 virus, no direct data have as yet been obtained, though it has been suggested that activation of the ANG-II signal pathway, which includes AT1R and phorbol-sensitive forms of PKC, must increase the proteolysis of the membrane-bound form of ACE2, thus influencing its involvement in the entry of virus into cells. However, clear conclusions on the interaction between ADAM17 activity and ACE2-mediated endocytosis of SARS-CoV-2 into cells will only be available after running the corresponding experiments [7].

Clinical Consequences of the Involvement of ACE2 in the Infection of Target Cells with SARS-CoV-2 Virus. The main contingent of patients suffering SARS-CoV-2 infection most severely are elderly people with atherosclerosis, hypertensive disease, ischemic heart disease, diabetes mellitus, and kidney and lung disease. Many of these 
diseases are due to derangements in the functioning of the renin-angiotensin system and are accompanied by compensatory or drug-induced changes in ACE2 expression [3-6, $56,57]$. As the membrane-bound form of ACE2 increases the penetration of SARS-CoV-2 into target cells, increases in the expression and quantity of this form of the enzyme in the membrane should promote SARS-CoV-2 infection and increases the severity of COVID-19. In this regard, we should note that ACE2 is expressed mainly in those tissues which are the main targets for SARS-CoV-2 and are the first to be infected - these are lung, kidney, intestine, blood vessel wall, brain, and testis cells [17, 18, 58-62].

Little time has passed since the outbreak of the pandemic, with the result that the questions of how the level of expression and activity of ACE2 in patients correlate with disease severity and how this affects the systemic treatment of patients with chronic cardiovascular system diseases and diabetes mellitus remain open. However, it is appropriate to express a number of important considerations on this theme now.

ACE2 activity decreases in diabetes mellitus, due largely to excess glycation of the enzyme molecule in the conditions of prolonged hyperglycemia typical of diabetic pathology, and this decrease is one of the primary causes of the predisposition of patients with diabetes to experience severe lung disease [63]. The decreases in ACE2 and ANG(1-7) activity in diabetes, these having anti-inflammatory and antioxidant actions, weaken the protection of lung tissue from viral infection, as demonstrated by the highly lethal avian influenza A (H5N1) [64]. There are good grounds for the view that the treatment of diabetes mellitus with a number of antidiabetic agents, including pioglitazone and liraglutide, increases ACE2 expression, as has been demonstrated in animal experiments $[3,65]$. Liraglutide, an analog of glucagon-like peptide 1 , given to both healthy rats and animals with experimental diabetes, significantly increased ACE2 expression in the lungs and increased the level of ANG-(1-7) circulating in the bloodstream [65]. Diabetes mellitus is usually accompanied by dysfunctions of the cardiovascular system, i.e., atherosclerosis and hypertension, which require specific therapy with ACE inhibitors, AT1R blockers, and statins. Use of these agents leads to significant increases in ACE2 expression, and this effect is seen in patients with cardiovascular diseases and in those without diabetic pathology $[3,8,57]$. This has been confirmed in animal experiments $[8,66-68]$. Administration of the ACE inhibitor lisinopril and the AT1R antagonist losartan to normotensive animals has been shown to increase ACE2 expression in the heart by factors of 4.7 and 2.8 , respectively [67]. Increases in ACE2 expression in heart muscle are also seen on administration of these drugs to Ren-2 hypertensive transgenic rats [68]. Treatment of both normotensive and hypertensive rats with lisinopril and losartan also increased ACE2 expression and activity in the kidneys, though to a lesser extent than in the heart $[68,69]$. Studies of the effects of different AT1R blockers in mice showed approximately two-fold increases in the quantity of ACE2 protein in heart muscle [70].

This creates a closed circuit. On the one hand, in the absence of treatment, ACE2 is generally decreased, which is accompanied by life-threatening functional disorders and reduced immunity, which is critical in conditions of COVID-19. On the other hand, treatment with certain antidiabetic and antihypertensive drugs leads to increases in ACE2 expression, which promotes the penetration of SARS-CoV-2 into cells. It is important to note that cells infected with SARS-CoV-2 by virus-induced endocytosis of enzyme ACE2 and its subsequent intracellular degradation have decreases in the quantity of functionally active ACE2 on target cell surfaces. This produces an even greater degradation of functional status in the lungs, cardiovascular system, and kidneys, and promotes the development of acute respiratory distress syndrome and acute heart failure in COVID-19 [8, 71]. In this regard, a number of investigators have suggested that during COVID-19 illness, patients taking systemic ACE inhibitors, AT1R blockers, and statins should discontinue these drugs and replace them with, for example, calcium channel blockers, or should use combined therapy [3, 57]. Despite the fact that the available literature lacks data on the effects of calcium channel blockers on ACE2 expression and activity, the possibility that decreases in the intracellular calcium ion concentration induced by these drugs leads to increased ACE2 expression cannot be excluded. This is because blockade of calcium signaling leads to inhibition of the same AT1R-dependent signal cascade which is suppressed by inhibition of AT1R or decreases in ANG-II production.

Significant levels of ACE2 expression have been demonstrated in several parts of the brain. On the one hand, this is yet another way of propagating SARS-CoV-2 infection and, on the other, it is the cause of a sharp reduction in the ACE2 level in the CNS due to its simultaneous internalization with virus particles into neurons [3]. High contents of virus particles and the associated transfer of degraded forms of ACE2 were found in the brainstem and the cranial nerves running from it $[72,73]$. This resulted in activation of apoptotic processes in these cells and their death, which led to impairments to the functioning of brain centers responsible for regulating arterial pressure and respiration. It should be noted that decreased ACE2 levels in the brainstem can also induce impairments to the tone of the sympathetic nervous system and exacerbate arterial hypertension [74-76] which, according to existing data, is typical of COVID-19.

Conclusions. Identification of the key roles of the renin-angiotensin system and ACE2 in host cells infected with SARS-CoV, the pathogen of atypical pneumonia, as well as SARS-CoV-2, the cause of the new COVID-19 pandemic, not only allows risk groups most susceptible to these diseases to be identified, but also provides a pathway to the de- 
velopment of novel approaches to creating antiviral drugs preventing coronavirus infection. Results from clinical observations of patients with COVID-19 show that the balance of the vasoconstrictor and vasodilator influences of peptides of the angiotensin family via different types of angiotensin receptors, the ratio of the activities of ACE and its functional antagonist ACE2, the pattern of membrane-bound proteases (TMPRSS2, ADAM17), which determine the fate of ACE2 and the ability of the virus to internalize into cells, not only influence the process of human infection with SARS-CoV-2 virus, but also largely determine the pathogenesis and outcome of COVID-19. Several approaches have now been proposed for creating drugs based on impairing the ability of the virus to enter cells - in particular, these are inhibitors of TMPRSS 2 protease, and also compounds able to prevent the effective interaction of ACE2 with viral S protein. These hopes are linked with generating soluble forms of ACE2, able to neutralize virus particles. However, this raises the risk of overproduction of ANG-(1-7), with patients developing hypotension. Measures directed to normalizing the renin-angiotensin system and optimizing the strategy of using drugs targeting ACE and AT1R are of no less interest, as imbalance in the ACE-ANG-II/ACE2-ANG-(1-7) system can have extremely pernicious consequences for patients with SARS-CoV-2 infection. All these approaches are in demand, and in combination with anti-SARS-CoV-2 vaccine development, which is also at its initial stages [77-79], may terminate the COVID-19 pandemic, which according to April 2020 data has already taken more than 180,000 human lives.

This work was supported by the State Assignment of the Institute of Evolutionary Physiology and Biochemistry, Russian Academy of Sciences (No. AAAA-A18-11801229 0427-7).

The author has no conflicts of interests.

\section{REFERENCES}

1. World Health Organization, WHO Director-General's Remarks at the Media Briefing on 2019-nCoV on 11 February 2020 (2020), https://www.who.int/dg/speeches/detail/who-director-general-s-remarks-at-the-media-briefing-on-2019-ncov-on-11-february-2020.

2. A. Wu, Y. Peng, B. Huang, et al., "Genome composition and divergence of the novel coronavirus (2019-nCoV) originating in China," Cell Host Microbe, 27, No. 3, 325e328 (2020), https://doi.org/10. 1016/j.chom.2020.02.001.

3. L. Fang, G. Karakiulakis, and M. Roth, "Are patients with hypertension and diabetes mellitus at increased risk for Covid-19 infection?" Lancet Respir. Med., 8, No. 4, e21 (2020), https://doi.org/10.1016/ S2213-2600(20)30116-8.

4. C. Huang, Y. Wang, X. Li, et al., "Clinical features of patients infected with 2019 novel coronavirus in Wuhan, China," Lancet, 395, 497-506 (2020), https://doi.org/10.1016/S0140-6736(20)30183-5.

5. Y. Y. Zheng, Y. T. Ma, J. Y. Zhang, and X. Xie, "COVID-19 and the cardiovascular system," Nat. Rev. Cardiol. (2020), https://doi.org/ 10.1038/s41569-020-0360-5.

6. P. Zhou, X. L. Yang, X. G. Wang, et al., "A pneumonia outbreak associated with a new coronavirus of probable bat origin," Nature, 579, No. 7798, 270-273 (2020), https://doi.org/10.1038/s41586-0202012-7.
7. A. H. J. Danser, M. Epstein, and D. Batlle, "Renin-angiotensin system blockers and the COVID-19 Pandemic: At present there is no evidence to abandon renin-angiotensin system blockers," Hypertension; HYPERTENSIONAHA12015082 (2020), https://doi.org/10. 1161/HYPERTENSIONAHA.120.15082.

8. A. M. South, D. Diz, and M. C. Chappell, "COVID-19, ACE2 and the cardiovascular consequences," Am. J. Physiol. Heart Circ. Physiol. (2020), https://doi.org/10.1152/ajpheart.00217.2020.

9. W. Li, M. J. Moore, N. Vasilieva, et al., "Angiotensin-converting enzyme 2 is a functional receptor for the SARS coronavirus," Nature, 426, 450-454 (2003), https://doi.org/10.1038/nature02145.

10. S. Matsuyama, N. Nagata, K. Shirato, et al., "Efficient activation of the severe acute respiratory syndrome coronavirus spike protein by the transmembrane protease TMPRSS2," J. Virol., 84, 12658-12664 (2010), https://doi.org/10.1128/JVI.01542-10.

11. A. Heurich, H. Hofmann-Winkler, S. Gierer, et al., "TMPRSS2 and ADAM17 cleave ACE2 differentially and only proteolysis by TMPRSS2 augments entry driven by the severe acute respiratory syndrome coronavirus spike protein," J. Virol., 88, No. 2, 1293-307 (2014), https://doi.org/10.1128/JVI.02202-13.

12. D. Batlle, J. Wysocki, and K. Satchell, "Soluble angiotensin-converting enzyme 2: a potential approach for coronavirus infection therapy?" Clin. Sci. (Lond.), 134, 543-545 (2020), https://doi.org/ 10.1042/CS20200163.

13. M. Hoffmann, H. Kleine-Weber, S. Schroeder, et al., "SARS-CoV-2 cell entry depends on ACE2 and TMPRSS2 and is blocked by a clinically proven protease inhibitor," Cell, pii: S0092-8674(20)30229-4 (2020), https://doi.org/10.1016/j.cell.2020.02.052.

14. H. Lu, L. A. Cassis, C. W. Kooi, and A. Daugherty, "Structure and functions of angiotensinogen," Hypertens. Res., 39, No. 7, 492-500 (2016), https://doi.org/10.1038/hr.2016.17.

15. C. Wu, H. Lu, L. A. Cassis, and A. Daugherty, "Molecular and pathophysiological features of angiotensinogen: a mini review," N. Am. J. Med. Sci. (Boston), 4, 183-190 (2011), https://doi.org/10. 7156/v4i4p183.

16. L. B. Arendse, A. H. J. Danser, M. Poglitsch, et al., "Novel therapeutic approaches targeting the renin-angiotensin system and associated peptides in hypertension and heart failure," Pharmacol. Rev., 71 No. 4, 539-570 (2019), https://doi.org/10.1124/pr.118.017129.

17. M. Donoghue, F. Hsieh, E. Baronas, et al., "A novel angiotensin-converting enzyme-related carboxypeptidase (ACE2) converts angiotensin I to angiotensin 1-9," Circ. Res., 87, E1-9 (2000), https://doi.org/10.1161/01.res.87.5.e1.

18. S. R. Tipnis, N. M. Hooper, R. Hyde, et al., "A human homolog of angiotensin-converting enzyme. Cloning and functional expression as a captopril-insensitive carboxypeptidase," J. Biol. Chem., 275, No. 43, 33238-33243 (2000), https://doi.org/10.1074/jbc.M002615200.

19. J. Varagic, S. Ahmad, S. Nagata, and C. M. Ferrario, "ACE2: angiotensin II/angiotensin-(1-7) balance in cardiac and renal injury," Curr.Hypertens.Rep., 16, No. 3, 420 (2014), https://doi.org/10.1007/ s11906-014-0420-5.

20. V. B. Patel, J. C. Zhong, M. B. Grant, and G. Y. Oudit, "Role of the ACE2/angiotensin 1-7 axis of the renin-angiotensin system in heart failure," Circ. Res., 118, No. 8, 1313-1326 (2016), https://doi. org/10.1161/CIRCRESAHA.116.307708.

21. D. W. Lambert, M. Yarski, F. J. Warner, et al., "Tumor necrosis factor-alpha convertase (ADAM17) mediates regulated ectodomain shedding of the severe-acute respiratory syndrome-coronavirus (SARS-CoV) receptor, angiotensin-converting enzyme-2 (ACE2)," J. Biol. Chem., 280, No. 34, 30113-30119 (2005), https://doi.org/10. 1074/jbc.M505111200.

22. J. Xu, S. Sriramula, H. Xia, et al., "Clinical relevance and role of neuronal AT1 receptors in ADAM17-mediated ACE2 shedding in neurogenic hypertension," Circ. Res., 121,43-55 (2017), https://doi. org/10.1161/CIRCRESAHA.116.310509. 
23. S. Mukerjee, H. Gao, J. Xu, et al., "ACE2 and ADAM17 interaction regulates the activity of presympathetic neurons," Hypertension, 74, No. 5, 1181-1191 (2019), https://doi.org/10.1161/ HYPERTENSIONAHA.119.13133.

24. F. Zunke and S. Rose-John, "The shedding protease ADAM17: Physiology and pathophysiology," Biochim. Biophys. Acta Mol.Cell Res., 1864, No. 11, Part B, 2059-2070 (2017), https://doi.org/10. 1016/j.bbamcr.2017.07.001.

25. E. Kaschina, P. Namsolleck, and T. Unger, "AT2 receptors in cardiovascular and renal diseases," Pharmacol. Res., 125, Part A, 39-47 (2017), https://doi.org/10.1016/j.phrs.2017.07.008.

26. P. Balakumar and G. Jagadeesh, "Structural determinants for binding, activation, and functional selectivity of the angiotensin AT1 receptor," J. Mol. Endocrinol., 53, No. 2, R71-92 (2014), https://doi. org/10.1530/JME-14-0125.

27. H. Zhang, G. W. Han, A. Batyuk, et al., "Structural basis for selectivity and diversity in angiotensin II receptors," Nature, 544, No. 7650, 327-332 (2017), https://doi.org/10.1038/nature22035.

28. S. AbdAlla, H. Lother, A. M. Abdel-tawab, and U. Quitterer, "The angiotensin II AT2 receptor is an AT1 receptor antagonist," J. Biol. Chem., 276, No. 43, 39721-39726 (2001), https://doi.org/10.1074/ jbc.M105253200.

29. S. Patel and T. Hussain, "Dimerization of AT2 and Mas receptors in control of blood pressure," Curr. Hypertens. Rep., 20, No. 5, 41 (2018), https://doi.org/10.1007/s11906-018-0845-3.

30. H. Akazawa, M. Yano, C. Yabumoto, et al., "Angiotensin II type 1 and type 2 receptor-induced cell signaling," Curr. Pharm. Des., 19, No. 17, 2988-2995 (2013), https://doi.org/10.2174/1381612811319 170003.

31. H. Zhang, H. Unal, R. Desnoyer, et al., "Structural basis for ligand recognition and functional selectivity at angiotensin receptor," J. Biol. Chem., 290, No. 49, 29127-29139 (2015), https://doi.org/10. 1074/jbc.M115.689000

32. G. Turu, A. Balla, and L. Hunyady, "The Role of $\beta$-arrestin proteins in organization of signaling and regulation of the AT1 angiotensin receptor," Front. Endocrinol. (Lausanne), 10, 519 (2019), https:// doi.org/10.3389/fendo.2019.00519.

33. T. Chiu, C. Santiskulvong, and E. Rozengurt, "ANG II stimulates PKC-dependent ERK activation, DNA synthesis, and cell division in intestinal epithelial cells," Am.J.Physiol.Gastrointest.Liver Physiol., 285, No. 1, G1-11 (2003), https://doi.org/10.1152/ajpgi.00419.2002.

34. J. Hines, S. J. Fluharty, and D. K. Yee, "Structural determinants for the activation mechanism of the angiotensin II type 1 receptor differ for phosphoinositide hydrolysis and mitogen-activated protein kinase pathways," Biochem. Pharmacol., 66, No. 2, 251-262 (2003), https://doi.org/10.1016/s0006-2952(03)00257-0.

35. P. Namsolleck, C. Recarti, S. Foulquier, et al., "AT2 Receptor and tissue injury: Therapeutic implications," Curr. Hypertens. Rep., 16, No. 2, 416 (2014), https://doi.org/10.1007/s11906-013-0416-6.

36. J. M. Saavedra and I. Armando, "Angiotensin II AT2 receptors contribute to regulate the sympathoadrenal and hormonal reaction to stress stimuli," Cell. Mol. Neurobiol., 38, No. 1, 85-108 (2018), https://doi.org/10.1007/s10571-017-0533-x.

37. D. M. Bennion, E. Haltigan, R. W. Regenhardt, et al., "Neuroprtective mechanisms of the ACE2-angiotensin-(1-7)-Mas axis in stroke," Curr. Hypertens. Rep., 17, No. 2, 3 (2015), https://doi.org/10.1007/ s11906-014-0512-2.

38. E. Böttcher, T. Matrosovich, M. Beyerle, et al., "Proteolytic activation of influenza viruses by serine proteases TMPRSS2 and HAT from human airway epithelium," J. Virol., 80, No. 19, 9896-9898 (2006), https://doi.org/10.1128/JVI.01118-06.

39. Y. Shirogane, M. Takeda, M. Iwasaki, et al., "Efficient multiplication of human metapneumovirus in Vero cells expressing the transmembrane serine protease TMPRSS2," J. Virol., 82, No. 17, 8942-8946 (2008), https://doi.org/10.1128/JVI.00676-08.
40. C. Chaipan, D. Kobasa, S. Bertram, et al., "Proteolytic activation of the 1918 influenza virus hemagglutinin," J. Virol., 83, No. 7, 32003211 (2009), https://doi.org/10.1128/JVI.02205-08.

41. B. Yun, Y. Zhang, Y. Liu, et al., "TMPRSS12 is an activating protease for subtype B avian metapneumovirus," J. Virol., 90, No. 24, 11231-11246 (2016), https://doi.org/10.1128/JVI.01567-16.

42. F. Li, W. Li, M. Farzan, and S. C. Harrison, "Structure of SARS coronavirus spike receptor-binding domain complexed with receptor," Science, 309, No. 5742, 1864-1868 (2005), https://doi.org/10. 1126/science.1116480.

43. Y. Wan, J. Shang, R. Graham, et al., Receptor recognition by novel coronavirus from Wuhan: an analysis based on decade-long structural studies of SARS," J. Virol., 94, No. 7, pii: JVI.00127-20 (2020), https://doi.org/10.1128/JVI.00127-20.

44. J. Lan, J. Ge, J. Yu, et al., "Structure of the SARS-CoV-2 spike receptor-binding domain bound to the ACE2 receptor," Nature (2020), https://doi.org/10.1038/s41586-020-2180-5.

45. J. Luan, Y. Lu, X. Jin, and L. Zhang, "Spike protein recognition of mammalian ACE2 predicts the host range and an optimized ACE2 for SARS-CoV-2 infection," Biochem. Biophys. Res. Commun., pii: S0006-291X(20)30526-X (2020), https://doi.org/10.1016/j.bbrc.2020. 03.047 .

46. S. Matsuyama, N. Nao, K. Shirato, et al., "Enhanced isolation of SARS-CoV-2 by TMPRSS2-expressing cells," Proc. Natl. Acad. Sci. USA, 117, No. 13, 7001-7003 (2020), https://doi.org/10.1073/pnas. 2002589117.

47. Y. Qiu, Y. B. Zhao, Q. Wang, et al., "Predicting the angiotensin converting enzyme 2 (ACE2) utilizing capability as the receptor of SARS-CoV-2," Microbes Infect., pii: S1286-4579(20)30049-6 (2020), https://doi.org/10.1016/j.micinf.2020.03.003.

48. J. Shang, G. Ye, K. Shi, et al., "Structural basis of receptor recognition by SARS-CoV-2," Nature, (2020), https://doi.org/10.1038/ s41586-020-2179-y.

49. R. Yan, Y. Zhang, Y. Li, et al., "Structural basis for the recognition of SARS-CoV-2 by full-length human ACE2," Science, 367, No. 6485, 1444-1448 (2020), https://doi.org/10.1126/science.abb2762.

50. N. Iwata-Yoshikawa, T. Okamura, Y. Shimizu, et al., "TMPRSS2 Contributes to virus spread and immunopathology in the airways of murine models after coronavirus infection," $J$. Virol., 93, No. 6, pii: e01815-18 (2019), https://doi.org/10.1128/JVI.01815-18.

51. G. Simmons, D. N. Gosalia, A. J. Rennekamp, et al., "Inhibitors of cathepsin L prevent severe acute respiratory syndrome coronavirus entry," Proc. Natl. Acad. Sci. USA, 102, No. 33, 11876-11881 (2005), https://doi.org/10.1073/pnas.0505577102.

52. M. Kawase, K. Shirato, L. van der Hoek, et al., "Simultaneous treatment of human bronchial epithelial cells with serine and cysteine protease inhibitors prevents severe acute respiratory syndrome coronavirus entry," J. Virol., 86, No. 12, 6537-6545 (2012), https://doi. org/10.1128/JVI.00094-12.

53. Y. Zhou, P. Vedantham, K. Lu, et al., "Protease inhibitors targeting coronavirus and filovirus entry," Antiviral Res., 116, 76-84 (2015), https://doi.org/10.1016/j.antiviral.2015.01.011.

54. J. E. Park, K. Li, A. Barlan, et al., "Proteolytic processing of middle east respiratory syndrome coronavirus spikes expands virus tropism," Proc. Natl.Acad.Sci. USA, 113, No. 43, 12262-12267 (2016), https://doi.org/10.1073/pnas.1608147113.

55. H. Kleine-Weber, M. T. Elzayat, M. Hoffmann, and S. Pöhlmann, "Functional analysis of potential cleavage sites in the MERScoronavirus spike protein," Sci.Rep., 8, No. 1, 16597 (2018), https:// doi.org/10.1038/s41598-018-34859-w.

56. J. H. Diaz, "Hypothesis: angiotensin-converting enzyme inhibitors and angiotensin receptor blockers may increase the risk of severe COVID-19," J. Travel Med., pii: taaa041 (2020), https://doi.org/10. 1093/jtm/taaa041. 
57. R. Pal and A. Bhansali, "COVID-19, diabetes mellitus and ACE2: The conundrum," Diabetes Res. Clin. Pract., 29, 108132 (2020), https://doi.org/10.1016/j.diabres.2020.108132.

58. C. M. Ferrario and J. Varagic, "The ANG-(1-7)/ACE2/mas axis in the regulation of nephron function," Am. J. Physiol., 298, F1297F1305 (2010), https://doi.org/10.1152/ajprenal.00110.2010.

59. M. Ohtsuki, S.-I. Morimoto, H. Izawa, et al., "Angiotensin converting enzyme 2 gene expression increased compensatory for left ventricular remodeling in patients with end-stage heart failure," Int. $J$. Cardiol., 145, No. 2, 333-334 (2010), https://doi.org/10.1016/j.ijcard. 2009.11.057.

60. S. H. Wong, R. N. Lui, and J. J. Sung, "Covid-19 and the digestive system,” J. Gastroenterol. Hepatol., (2020), https://doi.org/10.1111/ jgh.15047.

61. R. Kreutz, E. A. E. Algharably, M. Azizi, et al., "Hypertension, the renin-angiotensin system, and the risk of lower respiratory tract infections and lung injury: implications for COVID-19," Cardiovasc. Res.; pii: cvaa097 (2020), https://doi.org/10.1093/cvr/cvaa097.

62. L. Nicin, W. T. Abplanalp, H. Mellentin, et al., "Cell type-specific expression of the putative SARS-CoV-2 receptor ACE2 in human hearts," Eur. Heart J., pii: ehaa311 (2020), https://doi.org/10.1093/ eurheartj/ehaa311.

63. C. Tikellis and M. C. Thomas, "Angiotensin-converting enzyme 2 (ACE2) is a key modulator of the renin angiotensin system in health and disease," Int. J. Pept., 2012, 1-8 (2012), https://doi.org/10.1155/ 2012/256294.

64. Z. Zou, Y. Yan, Y. Shu, et al., "Angiotensin-converting enzyme 2 protects from lethal avian influenza A H5N1 infections," Nat. Commun., 5, 3594 (2014), https://doi.org/10.1038/ncomms4594.

65. M. Romaní-Pérez, V. Outeiriño-Iglesias, C. M. Moya, et al., "Activation of the GLP-1 receptor by liraglutide increases ACE2 expression, reversing right ventricle hypertrophy, and improving the production of SP-A and SP-B in the lungs of type 1 diabetes rats," Endocrinology, 156, No. 10,3559-3569 (2015), https://doi.org/10.1210/en.2014-1685.

66. L. Stanziola, L. J. Greene, and R. A. S. Santos, "Effect of chronic angiotensin converting enzyme inhibition on angiotensin I and bradykinin metabolism in rats," Am. J. Hypertens., 12, 1021-1029 (1999), https://doi.org/10.1016/S0895-7061(99)00090-4

67. C. M. Ferrario, J. Jessup, M. C. Chappell, et al., "Effect of angiotensin-converting enzyme inhibition and angiotensin II receptor blockers on cardiac angiotensin-converting enzyme 2," Circulation, 111, 2605-2610 (2005), https://doi.org/10.1161/CIRCULATIONAHA. 104.510461.

68. J. Jessup, P. E. Gallagher, D. B. Averill, et al., "Effect of angiotensin II blockade on a new congenic model of hypertension derived from the transgenic Ren-2 rat," Am. J. Physiol. Heart Circ., 291, No. 5, H2166-7H2172 (2006), https://doi.org/10.1152/ajpheart.00061.2006.
69. C. M. Ferrario, J. Jessup, P. E. Gallagher, et al., "Effect of renin-angiotensin system blockade on renal Angiotensin-(1-7)-forming enzymes, ACE-2 and receptors," Kidney Int., 68, No. 5, 2189-2196 (2005), https://doi.org/10.1111/j.1523-1755.2005.00675.x.

70. X. Wang, Y. Ye, H. Gong, et al., "The effects of different angiotensin II type 1 receptor blockers on the regulation of the ACE-AngIIAT1 and ACE2-Ang-(1-7)-Mas axes in pressure overload-induced cardiac remodeling in male mice," Mol. Cell Cardiol., 97, 180-190 (2016), https://doi.org/10.1016/j.yjmcc.2016.05.012.

71. C. Wu, X. Chen, Y. Cai, et al., "Risk factors associated with acute respiratory distress syndrome and death in patients with coronavirus disease 2019 pneumonia in Wuhan, China," JAMA Intern. Med. (2020), https://jamanetwork.com/journals/jamainternalmedicine/fullarticle/ 2763184.

72. Y. C. Li, W. Z. Bai, and T. Hashikawa, "The neuroinvasive potential of SARS-CoV2 may play a role in the respiratory failure of COVCID-19 patients," J. Med. Virol., 1, 1-4 (2020), https://doi.org/ 10.1002/jmv.25728.

73. L. Mao, M. Wang, S. Chen, et al., "Neurological manifestations of hospitalized patients with COVID-19 in Wuhan, China: a retrospective case series study," MedRExiv (2020), https://doi.org/10.1101/20 20.02.22.20026500.

74. D. I. Diz, M. A. Garcia-Espinosa, S. Gegick, et al., "Injections of angiotensin-converting enzyme 2 inhibitor MLN4760 into nucleus tractus solitarii reduce baroreceptor reflex sensitivity for heart rate control in rats," Exp. Physiol., 93, No. 5, 694-700 (2008), https:// doi.org/10.1113/expphysiol.2007.040261.

75. P. Xu, S. Sriramula, and E. Lazartigues, "ACE2/ANG-(1-7)/Mas pathway in the brain: the axis of good," Am.J.Physiol.Regul.Integr. Comp. Physiol., 300, No. 4, R804-R817 (2011), https://doi.org/10. 1152/ajpregu.00222.2010.

76. N. Alenina and M. Bader, "ACE2 in brain physiology and pathophysiology: Evidence from transgenic animal models," Neurochem. Res., 44, No. 6, 1323-1329 (2019), https://doi.org/10.1007/s11064018-2679-4.

77. W. H. Chen, U. Strych, P. J. Hotez, and M. E. Bottazzi, "The SARSCoV-2 vaccine pipeline: an overview," Curr. Top. Med. Rep., 3, 1-4 (2020), https://doi.org/10.1007/s40475-020-00201-6.

78. F. Amanat and F. Krammer, "SARS-CoV-2 vaccines: status report," Immunity, pii: S1074-7613(20)30120-5 (2020), https://doi.org/10. 1016/j.immuni.2020.03.007.

79. P. J. Hotez, M. E. Bottazzi, and D. B. Corry, "The potential role of Th17 immune responses in coronavirus immunopathology and vaccine-induced immune enhancement," Microbes Infect., pii: S1286-4579(20)30072-1 (2020), https://doi.org/10.1016/j.micinf. 2020.04.005. 